\title{
Does the oviparity-viviparity transition alter the partitioning of yolk in embryonic snakes?
}

\author{
Yan-Qing Wu', Yan-Fu Qu', Xue-Ji Wang ${ }^{1}$, Jian-Fang Gao² and Xiang $\mathrm{Ji}^{\text {1 }^{*}}$ (D)
}

\begin{abstract}
Background: The oviparity-viviparity transition is a major evolutionary event, likely altering the reproductive process of the organisms involved. Residual yolk, a portion of yolk remaining unutilized at hatching or birth as parental investment in care, has been investigated in many oviparous amniotes but remained largely unknown in viviparous species. Here, we used data from 20 (12 oviparous and 8 viviparous) species of snakes to see if the oviparity-viviparity transition alters the partitioning of yolk in embryonic snakes. We used ANCOVA to test whether offspring size, mass and components at hatching or birth differed between the sexes in each species. We used both ordinary least squares and phylogenetic generalized least squares regressions to test whether relationships between selected pairs of offspring components were significant. We used phylogenetic ANOVA to test whether offspring components differed between oviparous and viviparous species and, more specifically, the hypothesis that viviparous snakes invest more in the yolk as parental investment in embryogenesis to produce more well developed offspring that are larger in linear size.

Results: In none of the 20 species was sex a significant source of variation in any offspring component examined. Newborn viviparous snakes on average contained proportionally more water and, after accounting for body dry mass, had larger carcasses but smaller residual yolks than did newly hatched oviparous snakes. The rates at which carcass dry mass (CDM) and fat body dry mass (FDM) increased with residual yolk dry mass (YDM) did not differ between newborn oviparous and viviparous snakes. Neither CDM nor FDM differed between newborn oviparous and viviparous snakes after accounting for YDM.

Conclusions: Our results are not consistent with the hypothesis that the partitioning of yolk between embryonic and post-embryonic stages differs between snakes that differ in parity mode, but instead show that the partitioning of yolk in embryonic snakes is species-specific or phylogenetically related. We conclude that the oviparity-viviparity transition does not alter yolk partitioning in embryonic snakes.
\end{abstract}

Keywords: Offspring, Oviparity, Parity mode, Residual yolk, Snake, Viviparity, Yolk partitioning

\section{Background}

It is widespread and perhaps ubiquitous among invertebrates and non-mammalian vertebrates that embryos complete development without depleting the entire yolk reserve [1-5]. The yolk remaining unutilized at hatching (oviparous species) or birth (viviparous species), namely residual yolk, is internalized into the abdominal cavity of the offspring before emergence from the egg or mother. This portion of yolk can be subsequently metabolized

\footnotetext{
*Correspondence: xji@mail.hz.zj.cn

${ }^{1}$ Jiangsu Key Laboratory for Biodiversity and Biotechnology, College of Life

Sciences, Nanjing Normal University, Nanjing, Jiangsu 210023, China

Full list of author information is available at the end of the article
}

during the first days, weeks, or even months of life as a source of energy for maintenance metabolism and other essential activities prior to successful foraging [6-10] and contribute directly or indirectly to somatic tissue growth and thus linear growth ([11-13]; but see also [14-16]).

From previous studies on a wide range of vertebrate taxa we know the following. First, the size of residual yolk varies among species or taxa, among populations of the same species, among clutches of the same population or family, and even between the sexes of the same clutch $[5,9,17-19]$. For example, lizards $(0-12 \%$ of the body dry mass, with a mean of $5 \%$ ) generally have smaller residual yolks than do other reptiles (5-42\% of the body dry mass, 
with a mean of $21 \%)$ and birds $(21-56 \%$ of the body dry mass, with a mean of $34 \%$ ) studied so far (Additional file 1 : Table S1). Second, embryos cannot decide whether or not to use up the yolk or save some for later use, although residual yolk has a function to buffer the embryos from unpredictable environments and its quantity is affected by the environmental conditions experienced during embryonic development [20-22]. For example, high incubation or gestation temperatures and/or dry substrates often result in less developed offspring that characteristically have larger residual yolks but are smaller in linear size [23-27]. Third, fat-rich residual yolks better support post-hatch or post-natal activity or maintenance metabolism, whereas protein-rich residual yolks better support post-hatch growth $[22,28,29]$. Fourth, residual yolks are especially important for species where neonates and hatchlings have substantial energy expenditure before they begin to feed, including digging out of subterranean nests, long distance dispersal, or overwintering in nest cavities ([7, 9, 21, 30]; but see also $[16,31])$. Fifth, residual yolks seem to be essential in species that have less developed feeding appendages, organs, behaviors, gut flora and/or enzyme systems at hatching or birth $[2,10,32-34]$.

While residual yolk has been examined in a number of oviparous species, the occurrence, size and functional role of residual yolk in viviparous species remain almost unknown in non-mammalian amniote vertebrates. Oviparity is an ancestral mode of reproduction from which viviparity evolved independently $([35,36]$; but see also [37]). Viviparity has evolved in at least 115 lineages of squamate reptiles (lizards, snakes and amphisbaenians), and about one fifth of squamate reptiles are viviparous [38]. To our knowledge, however, the short-tailed pit-viper (Gloydius brevicaudus) is the only viviparous reptile for which the ratio of residual yolk dry mass to body dry mass has been reported [25]. The observation that the ratio and thus the relative size of residual yolk is far smaller in $G$. brevicaudus (3\% of the body dry mass [25]) than in any oviparous snake $(15-32 \%$ of the body dry mass, with an overall mean of 23\%; Additional file 1: Table S1) studied so far raises a question that forms the basis of this study: Does the evolutionary transition from oviparity to viviparity alter yolk partitioning in embryonic snakes? If so, one may hypothesize that the partitioning of yolk between embryonic (parental investment in embryogenesis, PIE) and post-embryonic (parental investment in care, PIC) stages should differ between oviparous and viviparous species, and in particular, viviparous species should produce more fully developed offspring that are larger in linear body size but have smaller residual yolks. Alternatively, it is possible that a smaller amount of yolk remaining at birth simply results from less yolk invested by viviparous species as PIC, perhaps owing to the increased maternal survival costs during gestation associated with carrying the yolk exceeding the need to produce a complete offspring [39, 40]. In the latter case, neonates of viviparous species should have smaller residual yolks when compared to newly hatched hatchlings of oviparous species of the same developmental condition.

Snakes are an ideal taxon for studying whether the developmental condition and yolk partitioning strategies are associated with parity mode because their viviparous species do not belong to any lineage that exhibits placentotrophy but rather all are lecithotrophic and yolk reserves support all energy demands during embryogenesis [41, 42]. Lecithotrophic viviparous species actually are similar to oviparous species in egg yolk and embryonic development and nutritional pattern [43]. Here, we used data collected from 20 (12 oviparous and 8 viviparous) species of snakes to address the above question.

\section{Methods}

Snakes were collected from three provinces in mainland China between 1998 and 2015, with four species from Guangxi in South China, 14 from Zhejiang in East China and two from Liaoning in Northeast China (Table 1). Detailed procedures for maintenance of gravid females and collection of eggs and newborn offspring, hatchlings (oviparous species) and neonates (viviparous species), have been described elsewhere [25, 44-47]. In brief, wildcaught gravid females were brought to our laboratory, where 1-3 females were housed in each wire (for terrestrial species) or glass (for aquatic species) cage until they laid eggs or gave birth to young. Cages were placed in an indoor animal holding facility where temperatures never varied outside the range of $24-30{ }^{\circ} \mathrm{C}$. Food [oriental weatherfish (Misgurnus anguillicaudatus), common toads (Bufo gargarizans), rice frogs (Fejervarya limnocharis), black-spotted frogs (Pelophylax nigromaculata), or house mice (Mus musculus)] and water were provided ad libitum. Eggs were collected and weighed less than $3 \mathrm{~h}$ post-laying. Eggs were either dissected to identify Zehr's [48] embryonic stage or incubated under multiple thermal conditions using Binder KB (Binder, Germany) or Shellab (Sheldon MFG Inc., USA) incubators. Hatchlings or neonates were collected, weighed, measured for snout-vent length (SVL) and tail length, and sexed (by manual eversion of hemipenes) less than $6 \mathrm{~h}$ emergence from the egg or mother. As extreme incubation temperatures often result in hatchlings that have either smaller (low temperatures) or larger (high temperatures) than usual residual yolks, only hatchlings from eggs incubated at temperatures moderate for each species were used in this study.

A total of 762 newborn offspring, 15-86 hatchlings (one or two individuals of different sexes from each clutch) from each oviparous species and 20-31 neonates (one or two individuals of different sexes from each litter) from each viviparous species were euthanized by freezing at $-20{ }^{\circ} \mathrm{C}$ on 


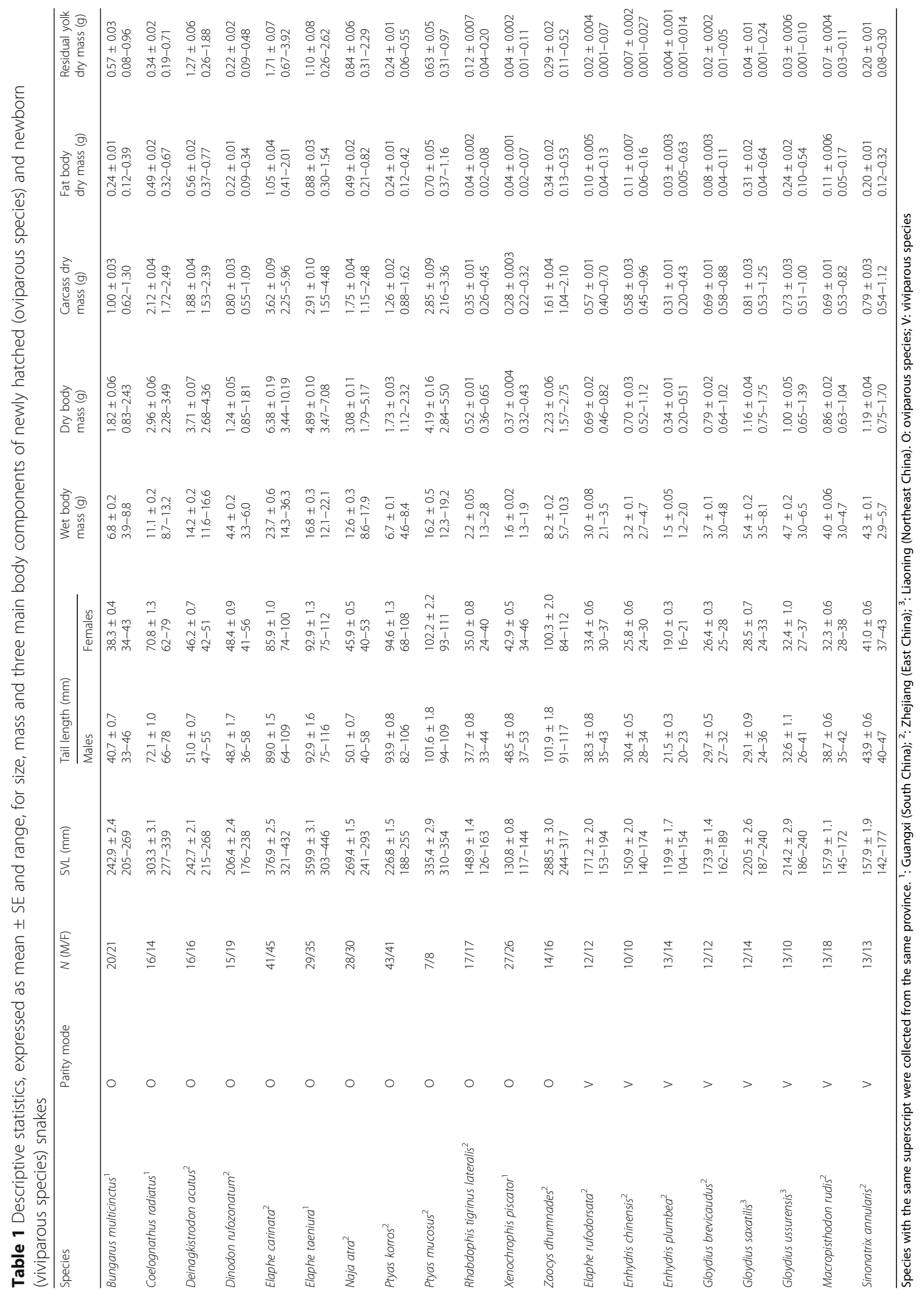


the day of hatching or birth. Frozen hatchlings and neonates were later thawed, dissected and separated into residual yolk, fat bodies and carcass. Freezing and thawing did not affect our ability to separate these components. The three components were dried to a constant mass in a $60{ }^{\circ} \mathrm{C}$ oven (Shanghai Senxin Ltd., China) for $48 \mathrm{~h}$ to obtain dry mass.

We used one-way ANCOVA with wet body mass or SVL (see below for note) as the covariate to test whether offspring size, mass and components (carcass, residual yolk and fat bodies) at hatching or birth differed between the sexes in each species. The same analysis was also used to test the parallelism of regression lines between oviparous and viviparous species. We used phylogenetic ANOVA to test whether proportional amounts of water, dry carcass, dry fat bodies and dry residual yolk differed between oviparous and viviparous species. Proportional data were arc-sine transformed prior to further analyses. We calculated residuals of carcass dry mass $(\mathrm{CDM}=$ hatchling dry mass - fat body dry mass - yolk dry mass) and fat body dry mass (FDM) against residual yolk dry mass (YDM) for each species, and then analyzed them using phylogenetic ANOVA to test whether CDM and FDM differed between oviparous and viviparous species with the same amount of YDM in R 3.3.0 [49] with the package GEIGER [50]. We used ordinary least squares (OLS) and phylogenetic generalized least squares (PGLS) regressions in R 3.3.0 with the packages RMS [51] and CAPER [52] to test whether relationships between selected pairs of offspring components were significant. The Akaike's Information Criterion (AIC) and likelihood-ratio test [53] were used to assess the adequacy of models tested. We performed phylogenetic ANOVA and PGLS to account for the non-independence of data due to the shared evolutionary history of species. To do that, we reconstructed a phylogeny for the 20 species using Mesquite 3.04 [54] based on the species-level phylogenetic relationships proposed by Pyron et al. $[55,56]$ and others $[57,58]$ for species of the families Colubridae, Elapidae, Natricinae and Viperidae (Fig. 1). We could not estimate individual branch lengths because sequences for reconstructing the phylogeny were either unavailable (Bungarus multicinctus and Gloydius ussurensis) or incomplete for some species (e.g. Coelognathus radiatus and Macropisthodon rudis). Consequently, we arbitrarily set branch lengths to 1 (Fig. 1), which is appropriate for a speciation model of evolution [59]. Phylogenetic signal was measured by Pagel's lambda $(\lambda)$ [60], which indicates the strength of the phylogenetic relationship. Lambda values of or near 0 indicate phylogenetic independence; values of or near 1 indicate that the variable is fully explained by evolutionary history and thus shows the maximal strength of phylogenetic signal [60]. Throughout this paper, values are presented as mean $\pm \mathrm{SE}$ and range, and the significance level is set at $P=0.05$.

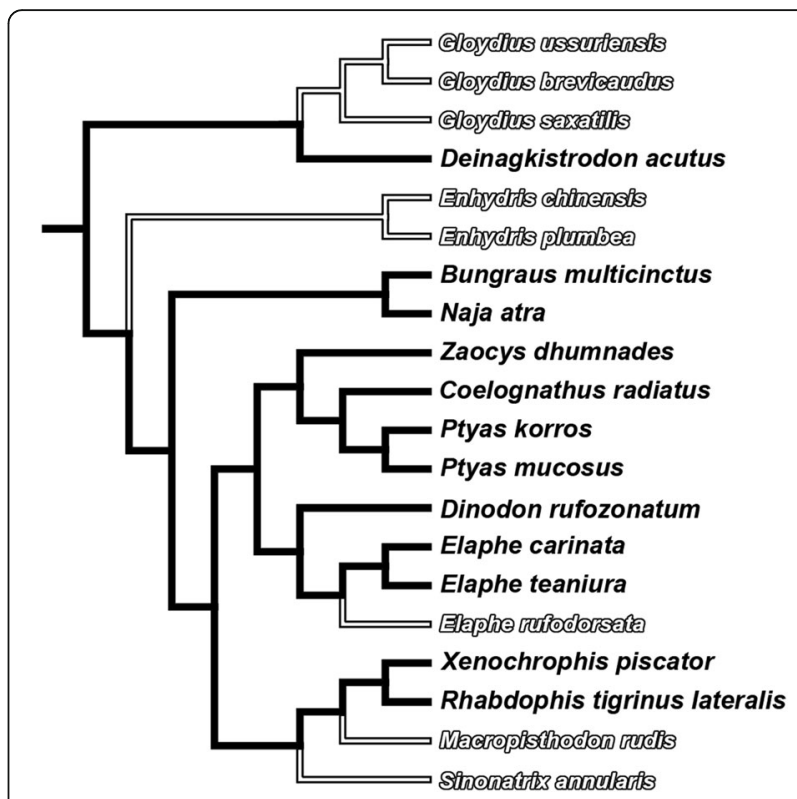

Fig. 1 The phylogeny of the 20 species of snakes used in this study. The topology was inferred from the proximate phylogenetic relationships at the species-level [55-58] and drawn using Mesquite 3.04 [54]. Oviparous species are in solid font, and viviparous species in hollow font

\section{Results}

Descriptive statistics for body size, mass and components of newborn offspring are given in Table 1. Two oviparous species (Deinagkistrodon acutus and Xenochrophis piscator) showed sexual size dimorphism at hatching, and in both species females were longer in SVL after accounting for wet body mass (ANCOVA: both $P<0.004$ ). Six oviparous (B. multicinctus, D. acutus, Elaphe carinata, Naja atra, Rhabdophis tigrinus lateralis and X. piscator) and six viviparous (Elaphe rufodorsata, Enhydris chinensis, Enhydris plumbea, Gloydius brevicaudus, M. rudis and Sinonatrix annularis) species showed sexual dimorphism in tail length at hatching or birth, and in all these species males were longer in tail length after accounting for SVL (ANCOVA: all $P<0.01)$. In none of the 20 species did we find that total body dry mass, CDM, FDM, or YDM differed between the sexes after accounting for wet body mass (ANCOVA: all $P>0.093$ ).

In the oviparous taxon species mean values for hatchling water contents ranged from $71 \%$ (Elaphe taeniura) to $77 \%$ (X. piscator) of body wet mass, with a mean of $74 \%$; in the viviparous taxa species mean values for neonate water contents ranged from $72 \%$ (S. annularis) to 79\% (En. chinensis, G. brevicaudus, G. saxatilis and M. rudis) of body wet mass, with a mean of $78 \%$ (Fig. 2). Newborn viviparous snakes on average contained proportionally more water than did newly hatched oviparous snakes (phylogenetic ANOVA: $F_{1,18}=17.28, P<$ $0.001)$. In the oviparous taxon species mean proportions 


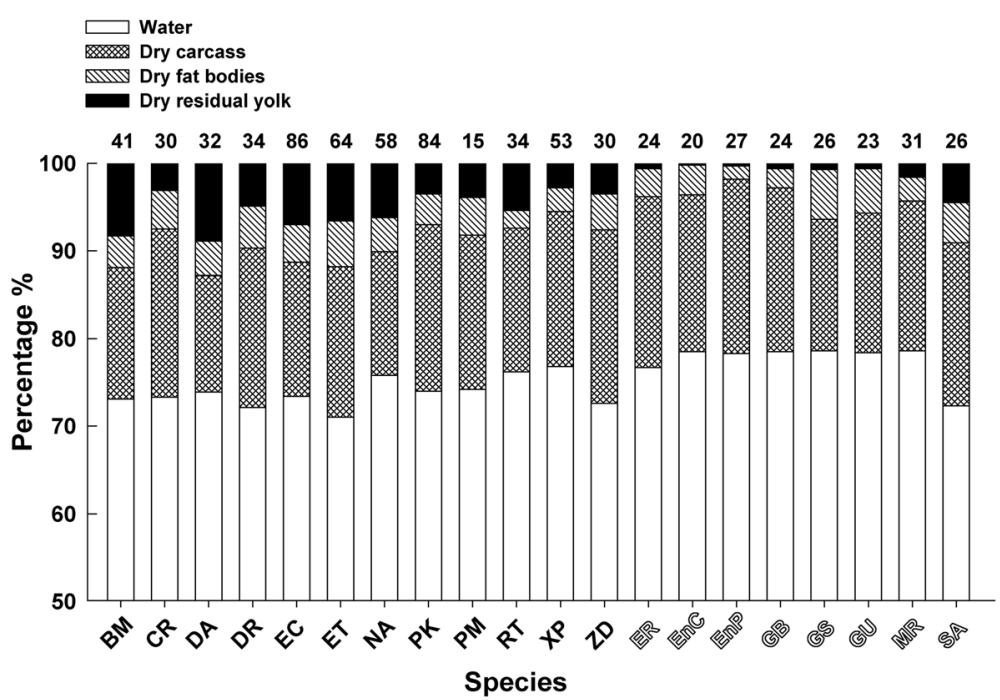

Fig. 2 Percentages of four major body components in newly hatched or newborn snakes. Numbers in the figure are sample sizes. Solid abbreviations represent oviparous species, and hollow abbreviations represent viviparous species. BM: B. multicinctus; CR: C. radiatus; DA: D. acutus; DR: D. rufozonatum; EC: E. carinata; ET: E. taeniura; NA: N. atra; PK: P. korros; PM: P. mucosus; RT: R. T. lateralis; XP: X. piscator; ZD: Z. dhumnades; ER: E. rufodorsata; EnC: En. chinensis; EnP: En. plumbea; GB: G. brevicaudus; GS: G. saxatilis; GU: G. ussurensis; MR: M. rudis; and SA: S. annularis

of CDM to hatchling dry mass ranged from $51 \%$ (D. acutus) to $76 \%$ (X. piscator), with a mean of $65 \%$; in the viviparous taxa species mean proportions of CDM to neonate dry mass ranged from 67\% (S. annularis) to 92\% (En. plumbea), with a mean of $80 \%$ (Fig. 2). Newborn viviparous snakes on average had relatively larger carcasses than did newly hatched oviparous snakes (phylogenetic ANOVA; $\left.F_{1,18}=8.99, P<0.01\right)$. Species mean proportions of FDM to hatchling dry mass ranged from 9\% (R. T. lateralis) to $18 \%$ (E. taeniura), with a mean of $15 \%$; species mean proportions of FDM to neonate dry mass ranged from 7\% (En. plumbea) to 27\% (G. saxatilis), with a mean of $16 \%$ (Fig. 2). Newborn viviparous snakes did not differ from newly hatched oviparous snakes in the relative size of fat bodies (phylogenetic ANOVA: $F_{1}$, ${ }_{18}=0.25, P=0.630$ ). Species mean proportions of YDM to hatchling dry mass ranged from $12 \%$ (C. radiatus) to $34 \%$ (D. acutus), with a mean of $20 \%$; species mean proportions of YDM to neonate dry mass ranged from $1 \%$ (En. chinensis and En. plumbea) to 16\% (S. annularis), with a mean of $5 \%$ (Fig. 2). Newborn viviparous snakes on average had relatively smaller residual yolks than did newly hatched oviparous snakes (phylogenetic ANOVA: $\left.F_{1,18}=25.29, P<0.0001\right)$.

Regression lines of CDM against YDM for oviparous and viviparous species were parallel (ANCOVA: $F_{1,16}=$ $0.006, P=0.938$ ), and so were regression lines of FDM against YDM (ANCOVA: $F_{1}, 16=0.003, P=0.956$ ). CDM (phylogenetic ANOVA: $F_{1,18}=0.83, P=0.375$ ) and FDM (phylogenetic ANOVA: $F_{1,18}=0.34, P=0.569$ ) did not differ between oviparous and viviparous species after accounting for YDM. Data pooled for oviparous and viviparous species showed that YDM explained $74 \%$ of variation in CDM, and $80 \%$ of variation in FDM (Fig. 3). The three offspring body components were positively related to each other, with all these relationships exhibiting strong phylogenetic signals (Table 2).

\section{Discussion}

While 12 species showed sexual dimorphism in SVL and/or tail length at hatching or birth, in none of the 20 species was sex a significant source of variation in the size of carcass, residual yolk, or fat bodies. This suggests that offspring sex is not related to maternal allocation of resources into egg yolk or the partitioning of yolk between PIE and PIC in snakes. Reptiles of different species or taxa show similar patterns of embryonic growth or yolk depletion in the course of embryonic development, which generally include three phases. The first phase is one of minimal transfer of energy and material from yolk to embryo, and the second and third phases are characterized by accelerated and, after an inflexion, decelerated embryonic growth or yolk depletion [61-65]. However, as we observed in this study (Fig. 2), yolk allocation strategies and thus proportions of yolk allocated to produce either larger offspring with smaller yolk reserves or smaller offspring with larger amounts of residual yolk may vary considerably among species [10, 66-69]. Natural selection for a given level of PIC is essentially influenced by the feeding ability of newborns or the period when they have a negative energy balance $[7,9,21,30]$. Thus, while larger residual yolks would provide sustenance for longer periods and better 


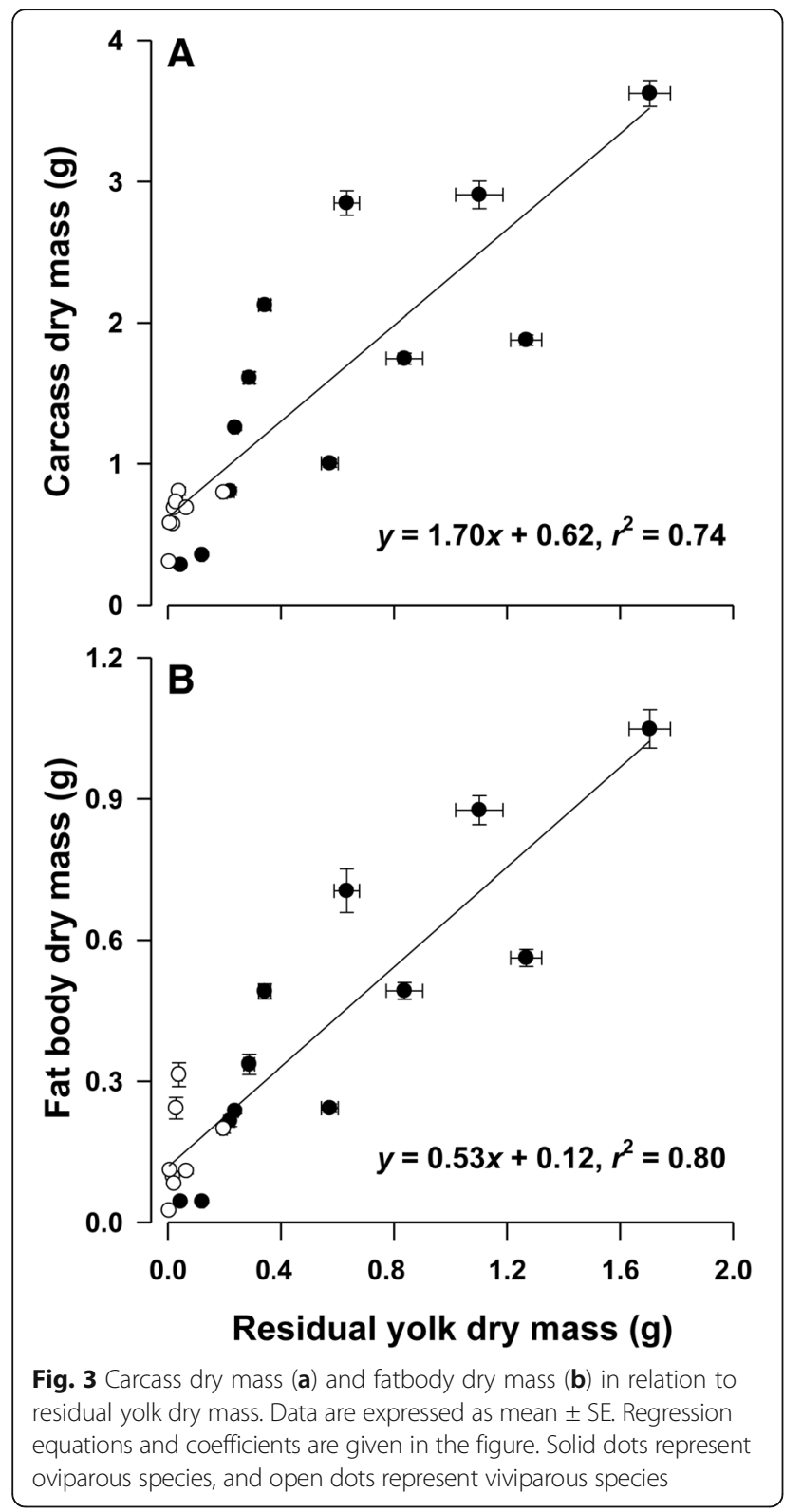

support early growth, larger offspring with smaller yolk reserves could be favored when resources are abundant or selection for high performance is strong [21, 22, 69, 70].

The first 1-3 weeks of life for oviparous snakes represent a period of time when they do not eat but often become even more fully developed as the consequence of early growth achieved by the post-hatching transfer of energy and material from residual yolk to carcass [44, 66-68]. Previous studies on several species of oviparous snakes including Dinodon rufozonatum [71], E. carinata [67], E. taeniura [68] and Ptyas korros [66] consistently show that more fully developed hatchlings are longer, have smaller residual yolks, and hold more water largely due to metabolic water production accompanied by yolk depletion. Here, we found that newborn viviparous snakes on average contained proportionally more water and, after accounting for body dry mass, they had larger carcasses but smaller residual yolks than did newly hatched oviparous snakes (Fig. 2). These findings suggest that viviparous snakes generally produce more fully developed offspring than oviparous snakes, but they do not support the hypothesis that the partitioning of yolk between PIE and PIC differs between snakes with different parity modes for two reasons. First, the partitioning of yolk between PIE and PIC and the degree of development at hatching or birth vary considerably among species in both oviparous and viviparous snakes (Fig. 2). This suggests that yolk partitioning in embryonic snakes is unlikely to be associated with parity mode but rather to be speciesspecific or phylogenetically related. In four aquatic viviparous snakes, for example, the relative size of carcass at birth was much smaller in $S$. annularis than in the other three species (E. rufodorsata, En. chinensis and En. plumbea; 67\% versus $83-91 \%$ of the body dry mass), whereas the reverse occurred for the relative size of residual yolk (16\% versus $1-3 \%$ of the body dry mass; Fig. 2). Second, viviparous snakes do not always produce more fully developed offspring with larger carcasses and smaller residual yolks. Sinonatrix annularis also offers an example, as its relative size of carcass was smaller than a half of 12 oviparous

Table 2 Parameters of regressions between each pair of three main body components (dry carcass, dry residual yolk and dry fat bodies) estimated with ordinary least squares (OLS) and phylogenetic generalized least squares (PGLS) regression models

\begin{tabular}{|c|c|c|c|c|c|c|c|c|c|}
\hline Models & $N$ & Slope & Elevation & $r^{2}$ & In likelihood & $\mathrm{AlC}$ & $\lambda$ & $F_{1,18}$ & $P$-value \\
\hline \multicolumn{10}{|l|}{ OLS regression model } \\
\hline Carcass vs Residual yolk & 20 & $1.70 \pm 0.23$ & $0.62 \pm 0.14$ & 0.74 & -13.43 & 32.86 & & 52.34 & $<0.0001$ \\
\hline Fat bodies vs Residual yolk & 20 & $0.53 \pm 0.06$ & $0.12 \pm 0.04$ & 0.80 & 13.27 & -20.55 & & 73.84 & $<0.0001$ \\
\hline Carcass vs Fat bodies & 20 & $3.25 \pm 0.16$ & $0.23 \pm 0.07$ & 0.96 & 4.16 & -2.33 & & 390.63 & $<0.0001$ \\
\hline \multicolumn{10}{|l|}{ PGLS regression model } \\
\hline Carcass vs Residual yolk & 20 & $1.50 \pm 0.19$ & $0.39 \pm 0.31$ & 0.76 & $-8.50^{\mathrm{a}}$ & 25.00 & 0.84 & 59.90 & $<0.0001$ \\
\hline Fat bodies vs Residual yolk & 20 & $0.46 \pm 0.06$ & $0.10 \pm 0.07$ & 0.79 & 15.01 & -22.03 & 0.54 & 59.52 & $<0.0001$ \\
\hline Carcass vs Fat bodies & 20 & $3.13 \pm 0.17$ & $0.16 \pm 0.13$ & 0.95 & $7.26^{\mathrm{a}}$ & -6.53 & 0.75 & 353.10 & $<0.0001$ \\
\hline
\end{tabular}

Models with a superscript of ${ }^{a}$ are significantly better than their alternate OLS or PGLS models 
species studied herein, including $C$. radiatus, $P$. korros, Ptyas mucosus, R. T. lateralis, $X$. piscator and Zaocys dhumnades $(67 \%$ versus $68-76 \%$ of the body dry mass; Fig. 2).

Given equal offspring mass, residual yolk mass is inversely related to yolk-free offspring mass ([61-65, 72]; but see also [22]). Here, we found in both oviparous and viviparous snakes that greater residual yolk mass occurred in species that produced larger offspring with larger carcasses and fat bodies and thus were heavier in yolk-free mass (Fig. 3). We also found that oviparous and viviparous snakes displayed the same rates at which CDM and FDM increased with YDM (Fig. 3) and that neither CDM nor FDM differed between newborn oviparous and viviparous snakes after accounting for YDM. These findings, when coupled with strong phylogenetic signals in all relationships between selected pairs of body components, allow us to conclude that the partitioning of yolk in embryonic snakes and the level of residual yolks or PIC are not associated with parity mode, but are instead species-specific or phylogenetically related.

Why do some snakes produce more fully developed offspring that emerge from the egg or mother later whereas others do not? To answer this question, we need to make a cost-benefit assessment. Snakes emerging earlier from the egg or mother have shorter lengths of embryonic development and larger residual yolks. For example, the mean incubation length at any temperature across the range within which embryonic development can take place is shorter in E. carinata (YDM accounting for $27 \%$ of total dry mass) than in E. taeniura (YDM accounting for $22 \%$ of total dry mass) [73, 74]. The benefit to an individual emerging immediately after completion of embryonic development is to initiate locomotion, feeding and growth toward maturity as soon as possible. This benefit is especially important for oviparous reptiles where parental care, if present, is quite limited $[75,76]$. The benefit of delayed emergence from the egg or mother is the proven sanctuary offered during a period of time when the benefit mentioned above is likely to be outweighed by predation or by mortality resulting from unfavorable environmental conditions [77, 78]. Potential costs of remaining in the egg or mother after completion of embryonic development is the increased mortality as a result of prolonged exposure of eggs or mothers to predators and other hostile factors, whereas potential costs of immediate emergence result primarily from high probabilities that newborns will encounter unfavorable situations such as earlier exposure to predators, drying up aquatic habitats or the onset of winter. These risks, when coupled with the lack of feeding ability in the first days or weeks of life, would result in immediate emergence being disadvantageous unless the disadvantages of remaining in the nest or mother were even higher. Delayed emergence is recognized as an adaptive trait for freshwater turtles where young individuals leaving the nest at the time of hatching might encounter inhospitable environmental conditions [77-79]. Unfortunately, parallel work on snakes has yet to be conducted. However, if delayed emergence is also adaptive for snakes, we predict that it will be most likely to occur in species that live in unpredictable and inhospitable environments with high levels of uncertainty about the cost-benefit of emergence. Future work could usefully investigate more lineages of reptiles with both oviparous and viviparous species also in a phylogenetic context to test this prediction.

\section{Conclusions}

Oviparity and viviparity are two distinct modes of reproduction that entail both advantages and disadvantages, of which each may differ in their applicability to particular species. Advantages and disadvantages associated with oviparity cannot be found in viviparity, and vice versa. However, oviparous and viviparous reptiles are common in that prolonged embryonic development increases not only energetic costs but also survival costs due to prolonged exposure of eggs or pregnant females to hostile biotic (such as predators and pathogenic microbes) and abiotic (such as lethal thermal and/or hydric extremes) factors. Our results are not consistent with the hypothesis that viviparous snakes have smaller amounts of residual yolk because they invest more in the yolk as PIE to produce more well developed offspring that are larger in linear size. Also, our results are not consistent with an alternative possibility that viviparous snakes have smaller amounts of residual yolk because they invest less in the yolk as PIC to reduce survival costs during gestation associated with carrying the yolk exceeding the need to produce a complete offspring. Instead, our results show that the partitioning of yolk in embryonic snakes is species-specific or phylogenetically related, thus allowing us to conclude that the oviparity-viviparity transition does not alter yolk partitioning in embryonic snakes.

\section{Additional file}

Additional file 1: Table S1. Non-mammalian amniotes for which data on the ratio of residual yolk dry mass to total hatchling dry mass have been available. O: oviparity; V: viviparity. (DOC 155 kb)

\section{Abbreviations \\ CDM: Carcass dry mass (= hatchling dry mass - fat body dry mass - yolk dry mass); FDM: Fat body dry mass; PIC: Parental investment in care; PIE: Parental investment in embryogenesis; SVL: Snout-vent length; YDM: Yolk dry mass}

Acknowledgments

We thank Yun Dai, Jue-Qing Du, Wei-Guo Du, Rui-Bin Hu, Long-Hui Lin, Zhi-Hua Lin, Hong-Liang Lu, Xue-Feng Xu and Yong-Pu Zhang for help in the laboratory, feeding and/or caring for snakes. 


\section{Funding}

This work was supported by grants from the National Natural Science Foundation of China to XJ $(31,272,294,30,770,378,30,370,229$ and $39,770,125)$ and YFQ (31770443), Natural Science Foundation of Zhejiang Province (RC97019 and Z3090461) to XJ, Priority Academic Program Development of Jiangsu Higher Education Institutions to XJ, and Postgraduate Innovative Training Program (KYLX_0716) of Nanjing Normal University to YQW. The funders had no role in the study design, data collection and analysis, decision to publish, or preparation of the manuscript.

\section{Availability of data and materials}

The datasets supporting the conclusions of this article are included within the article and its Additional file 1.

\section{Authors' contributions}

YQW and XJ conceived and designed the study. YQW, YFQ, XJW, JFG, and XJ conducted fieldwork. YQW, YFQ, XJW, and JFG conducted laboratory work and data analysis. YQW and XJ wrote the manuscript. All authors reviewed and contributed to editing of the manuscript and approved of its final publication.

\section{Ethics approval and consent to participate}

This study was conducted in accordance to the current Chinese laws on animal welfare and research, and approved by the Animal Research Ethics Committees of Nanjing Normal University and Hangzhou Normal University. The snakes in this study were collected under the permits issued by the Provincial Forestry Departments of Guangxi, Liaoning and Zhejiang.

\section{Consent for publication}

Not applicable.

\section{Competing interests}

The authors declare that they have no competing interests.

\section{Publisher's Note}

Springer Nature remains neutral with regard to jurisdictional claims in published maps and institutional affiliations.

\section{Author details}

${ }^{1}$ Jiangsu Key Laboratory for Biodiversity and Biotechnology, College of Life Sciences, Nanjing Normal University, Nanjing, Jiangsu 210023, China. ${ }^{2}$ Hangzhou Key Laboratory for Animal Adaptation and Evolution, College of Life and Environmental Sciences, Hangzhou Normal University, Hangzhou, Zhejiang 310036, China.

Received: 18 July 2017 Accepted: 17 November 2017

Published online: 29 November 2017

\section{References}

1. Congdon JD, Gibbons JW. Posthatching yolk reserves in hatchling American alligators. Herpetologica. 1989;45:305-9.

2. Gouiden CE, Henry L, Berrigan D. Egg size, postembryonic yolk, and survival ability. Oecologia. 1987;72:28-31.

3. Kamler $\mathrm{E}$, Keckeis $\mathrm{H}$, Bauer-Nemeschkal E. Temperature-induced changes of survival, development and yolk partitioning in Chondrostoma nasus. J Fish Biol. 1998;53:658-82

4. Kaplan RH. Ontogenetic energetics in Ambystoma. Physiol Zool. 1980;53:43-56.

5. Koláčková M, Prokůpková L, Albrecht T, Hořák D. Incubation temperature influences trade-off between structural size and energy reserves in mallard hatchlings. Physiol Biochem Zool. 2015;88:1-10.

6. Ewert MA. Cold torpor, diapause, delayed hatching and aestivation in reptiles and birds. In: Deeming DC, Ferguson MWJ, editors. Egg incubation: its effects on embryonic development in birds and reptiles. New York: Cambridge University Press; 1991. p. 173-91.

7. Lance VA, Morafka DJ. Post natal lecithotroph: a new age class in the ontogeny of reptiles. Herpetol Monogr. 2001:15:124-34

8. Sinervo B. The evolution of maternal investment in lizards: an experimental and comparative analysis of egg size and its effects on offspring performance. Evolution. 1990:44:279-94.

9. Spencer RJ, Janzen FJ. A novel hypothesis for the adaptive maintenance of environmental sex determination in a turtle. Proc R Soc B. 2014;281:20140831.
10. Troyer K. Posthatching yolk energy in a lizard: utilization pattern and interclutch variation. Oecologia. 1983:58:340-4

11. Filoramo $\mathrm{NI}$, Janzen FJ. Effects of hydric conditions during incubation on overwintering hatchlings of the red-eared slider turtle (Trachemys scripta elegans). J Herpetol. 1999;33:29-35.

12. Pandav BN, Shanbhag BA, Saidapur SK. Functional significance of posthatching residual yolk in the lizard, Calotes versicolor. J Herpetol. 2006;40:385-7.

13. Troyer K. Posthatching yolk in a lizard: internalization and contribution to growth. J Herpetol. 1987;21:102-6.

14. Giordano M, Groothuis TGG, Tschirren B. Interactions between prenatal maternal effects and posthatching conditions in a wild bird population. Behav Ecol. 2014;25:1459-66.

15. Murakami $\mathrm{H}$, Akiba $Y$, Horiguchi M. Growth and utilization of nutrients in newly-hatched chick with or without removal of residual yolk. Growth Dev Aging. 1992:56:75-84.

16. Radder RS, Warner DA, Cuervo JJ, Shine R. The functional significance of residual yolk in hatchling lizards Amphibolurus muricatus (Agamidae). Funct Ecol. 2007;21:302-9.

17. Allsteadt J, Lang JW. Incubation temperature affects body size and energy reserves of hatchling American alligators (Alligator mississippiensis). Physiol Zool. 1995:68:76-97.

18. Ji X, Gao JF, Han J. Phenotypic responses of hatchlings to constant versus fluctuating incubation temperatures in the multi-banded krait, Bungarus multicintus (Elapidae). Zool Sci. 2007;24:384-90.

19. Ji X, Huang HY, Hu XZ, Du WG. Geographic variation in female reproductive characteristics and egg incubation of Eumeces chinensis. Chin J Appl Ecol. 2002;13:680-4.

20. Lee TN, Plummer MV, Mills NE. Use of posthatching yolk and external forage to maximize early growth in Apalone mutica hatchlings. J Herpetol. 2007;41: 492-500.

21. Pezaro N, Doody JS, Green B, Thompson MB. Hatching and residual yolk internalization in lizards: evolution, function and fate of the amnion. Evol Dev. 2013;15:87-95.

22. Van Dyke JU, Plummer MV, Beaupre SJ. Residual yolk energetics and postnatal shell growth in smooth softshell turtles, Apalone mutica. Comp Biochem Physiol A. 2011:158:37-46.

23. Booth DT, Astill K. Incubation temperature, energy expenditure and hatchling size in the green turtle (Chelonia mydas), a species with temperature-sensitive sex determination. Austr J Zool. 2001;49:389-96.

24. Congdon JD, Gibbons JW. Turtle eggs: their ecology and evolution. In: Gibbons JW, editor. Life history and ecology of the slider turtle. Washington, DC: Smithsonian Institution Press; 1990. p. 109-23.

25. Gao JF, Qu YF, Luo LG, Ji X. Evolution of reptilian viviparity: a test of the maternal manipulation hypothesis in a temperate snake, Gloydius brevicaudus (Viperidae). Zool Sci. 2010;27:248-55.

26. Packard GC, Packard MJ, Miller K, Boardman TJ. Effects of temperature and moisture during incubation on carcass composition of hatchling snapping turtles (Chelydra serpentina). J Comp Physiol B. 1988;158:117-25.

27. Warner DA, Moody MA, Telemeco RS, Kolbe JJ. Egg environments have large effects on embryonic development, but have minima consequences for hatchling phenotypes in an invasive lizard. Biol J Linn Soc. 2012;105:25-41.

28. Alava JJ, Keller JM, Kucklick JR, Wyneken J, Crowder L, Scott Gl. Loggerhead sea turtle (Caretta caretta) egg yolk concentrations of persistent organic pollutants and lipid increase during the last stage of embryonic development. Sci Total Environ. 2006;367:170-81.

29. Carpentier AS, Booth DT, Arthur KE, Limpus CJ. Stable isotope relationships between mothers, eggs and hatchlings in loggerhead sea turtles Caretta caretta. Mar Biol. 2015;162:783-97.

30. Nagle RD, Plummer MV, Congdon JD, Fischer FU. Parental investment, embryo growth, and hatchling lipid reserves in softshell turtles (Apalone mutica) from Arkansas. Herpetologica. 2003;59:145-54.

31. Muir TJ, Dishong BD, Lee RE, Costanzo JP. Energy use and management of energy reserves in hatchling turtles (Chrysemys picta) exposed to variable winter conditions. J Therm Biol. 2013;38:324-30.

32. Benson AA, Lee RF. The role of wax in oceanic food chains. Sci Am. 1975; 232:77-86.

33. Blaxter JHS. Development: eggs and larvae. In: Hoar WS, Randall DJ, editors. Fish physiology, vol. 3. New York: Academic Press; 1969. p. 177-252.

34. Troyer K. Transfer of fermentative microbes between generations in a herbivorous lizard. Science. 1982;216:540-2. 
35. Blackburn DG. Are viviparity and egg-guarding evolutionarily labile? Herpetologica. 1999;55:556-73.

36. Griffith OW, Blackburn DG, Brandley MC, Van Dyke JU, Whittington CM, Thompson MB. Ancestral state reconstructions require biological evidence to test evolutionary hypotheses: a case study examining the evolution of reproductive mode in squamate reptiles. J Exp Zool B. 2015;324:493-503.

37. Pyron R, Burbrink FT. Early origin of viviparity and multiple reversions to oviparity in squamate reptiles. Ecol Lett. 2014;17:13-21.

38. Blackburn DG. Evolution of vertebrate viviparity and specializations for fetal nutrition: a quantitative and qualitative analysis. J Morphol. 2015;276:961-90.

39. Itonaga K, Jones SM, Wapstra E. Do gravid females become selfish? Female allocation of energy during gestation. Physiol Biochem Zool. 2012;85:231-42.

40. Crespi B, Semeniuk C. Parent-offspring conflict in the evolution of vertebrate reproductive mode. Am Nat. 2004;163:635-53.

41. Blackburn DG, Stewart JR. Viviparity and placentation in snakes. In: Aldrich $\mathrm{RD}$, Sever DM, editors. Reproductive biology and phylogeny of snakes. Enfield: science publishers; 2011. p. 119-81.

42. Thompson MB, Speake BK. Energy and nutrient utilisation by embryonic reptiles. Comp Biochem Physiol A. 2003;133:529-38.

43. Stewart JR, Thompson MB. Evolution of placentation among squamate reptiles: recent research and future directions. Comp Biochem Physiol A. 2000;127:411-31

44. Ji X, Du WG, Xu XF. Influences of thermal and hydric environments on incubating eggs and resultant hatchlings in a colubrid snake (Xenochrophis piscator). Acta Zool Sin. 2001;47:45-52.

45. Ji X, Du WG, Qu YF, Lin LH. Nonlinear continuum of egg size-number tradeoffs in a snake: is egg-size variation fitness related. Oecologia. 2009;159:689-96.

46. Lin LH, Mao F, Chen C, Ji X. Reproductive traits of the gray ratsnake Ptyas korros from three geographically distinct populations. Curr Zool. 2012;58:820-7.

47. Qu YF, Li H, Gao JF, Ji X. Geographical variation in reproductive traits and trade-offs between size and number of eggs in the king ratsnake, Elaphe carinata. Biol J Linn Soc. 2011:104:701-9.

48. Zehr DR. Stages in the normal development of the common garter snake, Thamnophis sirtalissirtalis. Copeia. 1962;1962:322-9.

49. R Development Core Team. R: A language and environment for statistical computing. Vienna, Austria: R Foundation for Statistical Computing. http://www.r-project.org; 2015.

50. Harmon LJ, Weir JT, Brock CD, Glor RE, Challenger WGEIGER. Investigating evolutionary radiations. Bioinformatics. 2008;24:129-31.

51. Harrell Jr FE Rms: regression modeling strategies. R package version 4.3-0. Available from: https://cran.r-project.org/web/packages/rms/index.html; 2015.

52. Orme D, Freckleton R, Thomas G, Petzoldt T, Fritz S, Isaac N, Pearse W. The caper package: comparative analysis of phylogenetics and evolution in $\mathrm{R}$. https://cran.r-project.org/web/packages/caper/index.html; 2013.

53. Warne RW, Charnov EL. Reproductive allometry and the size-number tradeoff for lizards. Am Nat. 2008;172:E80-98.

54. Maddison WP, Maddison DR. Mesquite: a modular system for evolutionary analysis. Version 3.04. http://mesquiteproject.org; 2015.

55. Pyron RA, Burbrink FT, Colli GR, de Oca ANM, Vitt LJ, Kuczynski CA, Wiens JJ. The phylogeny of advanced snakes (Colubroidea), with discovery of a new subfamily and comparison of support methods for likelihood trees. Mol Phylogenet Evol. 2011;58:329-42.

56. Pyron R, Burbrink FT, Wiens JJA. Phylogeny and revised classification of Squamata, including 4161 species of lizards and snakes. BMC Evol Biol. 2013;13:93.

57. Utiger U, Schätti B, Helfenberger $N$. The oriental colubrine genus Coelognathus Fitzinger, 1843 and classification of old and new world racers and ratsnakes (Reptilia, Squamata, Colubridae, Colubrinae). Russ J Herpetol. 2005:12:39-60.

58. Wang YQ, Zhou KY, Xu LS, Yang G, Xu GJ. The evolutionary relationships of several colubrid snakes suggested by sequences analysis of cyt $b$ gene fragment. Acta Zool Sin. 1999;45:332-8.

59. Martins EP, Garland T. Phylogenetic analyses of the correlated evolution of continuous characters: a simulation study. Evolution. 1991;45:534.

60. Pagel M. Inferring the historical patterns of biological evolution. Nature. 1999:401:877-84

61. Cai Y, Zhou T, Ji X. Embryonic growth and mobilization of energy and material in oviposited eggs of the red-necked keelback, Rhabdophis tigrinus lateralis. Comp Biochem Physiol A. 2007;147:57-63.

62. Du WG, Ji X, Xu WQ. Dynamics of material and energy during incubation in the soft-shelled turtle (Pelodiscus sinensis). Acta Zool Sin. 2001;47:371-5.
63. Lu HL, Hu RB, Ji X. Embryonic growth and mobilization of energy and material during incubation in the checkered keelback snake, Xenochrophis piscator. Comp Biochem Physiol A. 2009;152:214-8.

64. Shadrix CA, Crotzer DR, McKinney SL, Stewart JR. Embryonic growth and calcium mobilization in oviposited eggs of the scincid lizard, Eumeces fasciatus. Copeia. 1994;1994:493-8.

65. XF X, YL W, Zhang JL. Dynamics of material and energy during incubation in the grass lizards Takydromus septentrionalis. Acta Zool Sin. 2004;50:37-42.

66. Ji X, Sun PY. Embryonic use of energy and post-hatching yolk in the gray rat snake, Ptyas korros (Colubridae). Herpetol J. 2000;10:13-7.

67. Ji X, Sun PY, Fu SY, Zhang HS. Utilization of energy and nutrients in incubating eggs and post-hatching yolk in a colubrid snake, Elaphe carinata Herpetol J. 1997;7:7-12.

68. Ji X, Sun PY, Fu SY, Zhang HS. Utilization of egg energy and material during incubation and post-hatching yolk in a colubrid snake, Elaphe taeniura. Asiat Herpetol Res. 1999;8:53-9.

69. Radder RS, Shanbhag BA, Saidapur SK. Yolk partitioning in embryos of the lizard, Calotes versicolor: maximize body size or save energy for later use? J Exp Zool A. 2004;301:783-5.

70. Booth DT. Incubation of eggs of the Australian broad-shelled turtle, Chelodina expansa (Testudinata: Chelidae), at different temperatures: effects on pattern of oxygen consumption and hatchling morphology. Austr J Zool. 2000;48:369-78.

71. Ji X, Xu XF, Lin ZH. Influence of incubation temperature on characteristics of Dinodon rufozonatum (Reptilia: Colubridae) hatchlings, with comments on the function of residual yolk. Zool Res. 1999;20:342-6.

72. Thompson MB, Stewart JR. Embryonic metabolism and growth of the genus Eumeces. Comp Biochem Physiol A. 1997;118:647-54.

73. Du WG, Ji X. The effects of incubation temperature on hatching success, embryonic use of energy and hatchling morphology in the stripe-tailed ratsnake Elaphe taeniura. Asiat Herpetol Res. 2008;11:24-30.

74. Ji X, Du WG. The effects of thermal and hydric environments on hatching success, embryonic use of energy and hatchling traits in a colubrid snake, Elaphe carinata. Comp Biochem Physiol A. 2001;129:461-71.

75. Shine R. 1988. Parental care in reptiles. In: Gans C, Huey RB, editors. Biology of the Reptilia, vol. 16. New York: A. R. Liss; 1988. p. 275-330.

76. Shine R. Life-history evolution in reptiles. Annu Rev Ecol Evol Syst. 2005;36: 23-46.

77. Gibbons JW. A long-term perspective of delayed emergence (aka overwintering) in hatchling turtles: some they do and some they don't, and some you just can't tell. J Herpetol. 2013;47:203-14.

78. Lovich JE, Ernst CH, Ernst EM, Riley JL. A 21-year study of seasonal and interspecific variation of hatchling emergence in a Nearctic freshwater turtle community: to overwinter or not to overwinter. Herpetol Monogr. 2014;28: 93-109.

79. Riley JL, Tattersall GJ, Litzgus JD. Potential sources of intra-population variation in the overwintering strategy of painted turtle (Chrysemys picta) hatchlings. J Exp Biol. 2014;217:4147-83.

\section{Submit your next manuscript to BioMed Central and we will help you at every step:}

- We accept pre-submission inquiries

- Our selector tool helps you to find the most relevant journal

- We provide round the clock customer support

- Convenient online submission

- Thorough peer review

- Inclusion in PubMed and all major indexing services

- Maximum visibility for your research

Submit your manuscript at www.biomedcentral.com/submit
Biomed Central 